TRANSACTIONS OF THE

AMERICAN MATHEMATICAL SOCIETY

Volume 365, Number 10, October 2013, Pages 5579-5604

S 0002-9947(2013)05870-9

Article electronically published on March 12, 2013

\title{
RANDOM RIESZ ENERGIES ON COMPACT KÄHLER MANIFOLDS
}

\author{
RENJIE FENG AND STEVE ZELDITCH
}

\begin{abstract}
The expected Riesz energies $E_{\mu_{h}^{N}} \mathcal{E}_{s}$ of the zero sets of systems of independent Gaussian random polynomials of degree $N$ are determined asymptotically as the degree $N \rightarrow \infty$ in all dimensions and codimensions. The asymptotics are proved for sections of any positive line bundle over any compact Kähler manifold.
\end{abstract}

\section{INTRODUCTION}

A classical problem is to determine the optimal configurations of $N$ points $\left\{z^{1}, \ldots, z^{N}\right\}$ on the unit sphere $S^{2}$, i.e. configurations which minimize an energy function,

$$
\mathcal{E}_{s}\left(z^{1}, \cdots, z^{N}\right)=\sum_{i \neq j} G_{s}\left(z^{i}, z^{j}\right)
$$

for some kernel $G_{s}\left(z^{i}, z^{j}\right)$. The problem of minimizing energy is of interest on any compact Riemannian manifold, although it seems mainly to have been studied on the standard sphere. On a Riemannian manifold $(M, g)$ of dimension $2 m$, natural choices of the kernel $G_{s}(z, w)$ are the Riesz kernels,

$$
G_{s}(z, w)= \begin{cases}r_{g}(z, w)^{-s}, & 0<s<2 m, \\ -\log r_{g}(z, w), & s=0 .\end{cases}
$$

Here, $r_{g}(z, w)$ is the geodesic distance between $z, w$. The assumption $0<s<2 m$ ensures that the Riesz function $r_{g}(z, w)^{-s}$ is integrable. The minimal energy is denoted by

$$
\mathcal{E}_{s}(N)=\min _{\left\{z_{1}, \ldots, z_{N}\right\}} \mathcal{E}_{s}\left(z^{1}, \ldots, z^{N}\right) .
$$

For background on this problem, we refer to BBGKS, $\mathrm{HS}$.

Of course, it is a very demanding problem to determine the actual optimizers. Related problems are to find the asymtotics of the minimial energy (3) as $N \rightarrow \infty$ and to determine the properties of asymptotically minimizing configurations, which are sometimes called elliptic Fekete points. For instance, to minimize energy the points must be well separated and equidistributed with respect to the volume form.

This article is concerned with the expected energies $E_{\mu_{h}^{N}} \mathcal{E}_{s}$ of the zero sets of Gaussian random systems of holomorphic polynomials and their generalizations to any compact Kähler manifold. Thus, we do not study optimal configurations

Received by the editors January 11, 2012 and, in revised form, April 23, 2012.

2010 Mathematics Subject Classification. Primary 58J37, 32L81, 32A60, 60D05.

This research was partially supported by NSF grant \#DMS-0904252. 
but rather consider the separation and equidistribution properties of configurations defined by random zero sets as measured by the energies (1).

Our results generalize those of a recent pair of articles [Z, ABS] in the complex one dimensional case, which showed that the zeros of Gaussian random holomorphic polynomials of one complex variable are asymptotic energy minimizing configurations to a certain order in $N$. The Gaussian measures and results are reviewed in $\$ 1.2$ Such polynomials are holomorphic sections of $\mathcal{O}(N) \rightarrow \mathbb{C P}^{1}=S^{2}$, the $N$ th power of the hyperplane bundle over the Riemann sphere. It has been known for some time that in complex dimension one, the zeros of random polynomials of degree $N$ (or of any holomorphic sections) repel each other so that the nearest neighbors are almost always of order $\frac{1}{\sqrt{N}}$ apart. More precisely, the probability of pairs being $\frac{C}{\sqrt{N}}$ apart can be determined from the scaling asymptotics of the two-point correlation function of zeros in [H, BSZ1, BSZ2, BSZ3]. Furthermore, the 'empirical measure of zeros' defined by

$$
\mu_{z}=\frac{1}{N} \sum_{z: s^{N}(z)=0} \delta_{z}
$$

tends to the 'equilibrium measure' given by the Riemannian area form [SZ2]. The leading order asymptotics of the energy mainly reflect this repulsion and equidistribution. Repulsion and equidistribution are not sufficient to ensure that random zero point configurations asymptotically minimize energy, and indeed one would not expect a random configuration to do as well as a carefully chosen one. But in complex dimension one, random zero sets do surprisingly well (see \$1.2).

In this article, we consider the expected energies of the zero sets of a random full system of $m$ holomorphic polynomials on a complex projective space $\mathbb{C P}^{m}$ of any dimension. In fact, we consider the problem for sections of ample holomorphic line bundles $L \rightarrow M$ over general Kähler manifolds and, moreover, for systems of $k \leq m$ holomorphic sections. We refer to the case of a full system of $m$ polynomials in complex dimension $m$, whose simultaneous zero set is (almost surely) a discrete set of points, as the point case or equivalently as the discrete case.

In higher dimensions, we do not expect the complex zeros in the discrete case to asymptotically minimize energy. They do become equidistributed with respect to the equilibrium measure, but they are not well separated. As recalled in 22.4 . the scaling limits of the pair correlation function of zeros were calculated in BSZ1, BSZ2, BSZ3, in all dimensions and co-dimensions. The results show that, in the point case, the zeros almost ignore each other with only a slight repulsion at very close distances in dimension 2 , and they in fact attract or clump together in higher dimensions. The results of this article give a somewhat different perspective on the separation of the zeros as reflected by energy asymptotics, or more precisely on the distance of zeros from the discriminant variety of polynomial systems with a double root (see \$2.5).

Before stating our results on expected energies, let us review what is known about the minimum energies of configurations of points in the point case so that we can compare expected energies to minimal energies.

1.1. Minimal energies. First, we review results on $\mathbb{C P}^{1}=S^{2}$. We caution that different authors use slightly different normalizations of the radius of the sphere 
and the choice of Riesz kernels (2), and the coefficients of the asymptotic energies reflect these choices.

In $\mathrm{KS}, \mathrm{BBP}, \mathrm{RSZ}$, the authors consider the energy (1) with

$$
G_{s}(z, w)=\frac{1}{[z, w]^{s}}(s \neq 0) \text {; resp. } G_{0}(z, w)=\log \frac{1}{[z, w]} \quad(s=0) .
$$

Here, $[z, w]$ is the chordal distance between two points on $S^{2} \subset \mathbb{R}^{3}$ rather than the geodesic distance as in (2). They are related by $[z, w]=2 \sin \frac{r}{2}$ on the sphere of radius $\frac{1}{2}$ (i.e. area $\pi$ ). In $[\mathrm{KS}, \mathrm{W} 1, \mathrm{~W} 2$, it is proved (with the chordal distance) that

$$
\begin{cases}\mathcal{E}_{2}(N) \sim \frac{1}{8} N^{2} \log N, & s=2, \\ C_{1}(s) N^{1+\frac{s}{2}} \leq \mathcal{E}_{s}(N) \leq C_{2}(s) N^{1+\frac{s}{2}}, & s>2 \\ \frac{1}{2} V_{2}(s) N^{2}-C_{3}(s) N^{1+\frac{s}{2}} \leq \mathcal{E}_{s}(N) \leq \frac{1}{2} V_{2}(s) N^{2}-C_{4}(s) N^{1+\frac{s}{2}}, & s<2\end{cases}
$$

where all $C_{j}(s)$ and $V_{2}(s)$ are positive constants independent of $N$.

The asymptotic energy $\mathcal{E}_{0}(N)$ on the standard $S^{2}$ (again with the chordal distance) was further studied in [BBP, RSZ]. Theorem 3.1 of [RSZ] asserts that if $C_{N}$ is defined by

$$
\mathcal{E}_{0}(N)=-\frac{1}{4} \log \left(\frac{4}{e}\right) N^{2}-\frac{N}{4} \log N+C_{N} N,
$$

then for the standard $S^{2}$ one has

$$
-\frac{1}{4} \log \frac{\pi \sqrt{3}}{2}-\frac{\pi}{8 \sqrt{3}} \geq \limsup _{N \rightarrow \infty} C_{N} \geq \liminf _{N \rightarrow \infty} C_{N} \geq-\frac{1}{4} \log \left(\frac{\pi}{2}\left(1-e^{-a}\right)^{b}\right),
$$

for certain explicit constants $a, b$. But the precise value of $C_{N}$ is not known.

In the higher dimensional sphere $S^{m}$, results of [B, $\left.\mathrm{KS}\right]$ show that

$$
\begin{cases}C_{1}(s) N^{1+s / m} \leq \mathcal{E}_{s}(N) \leq C_{2}(s) N^{1+s / m}, & s>m, \\ \mathcal{E}_{m}(N) \sim \gamma_{m} N^{2} \log N, & s=m, \\ \frac{1}{2} V_{m}(s) N^{2}-C_{3}(s) N^{1+\frac{s}{m}} \leq \mathcal{E}_{s}(N) \leq \frac{1}{2} V_{m}(s) N^{2}-C_{4}(s) N^{1+\frac{s}{m}}, & s<m,\end{cases}
$$

where $V_{m}(s), \gamma_{m}$ and $C_{j}(s)$ are positive constants independent of $N$. Many further references and results can be found in BBGKS.

1.2. Expected energies of zeros of random polynomial systems. We now consider the energies of a random configuration of points which are defined as the zeros of systems of random polynomials (or sections). Let $(L, h) \rightarrow(M, \omega)$ be a positive holomorphic line bundle equipped with a Hermitian metric $h=e^{-\phi}(\phi$ is the Kähler potential). Let $H^{0}\left(M, L^{N}\right)$ be the space of the global holomorphic sections of the line bundle $L^{N}$; put $d_{N}:=\operatorname{dim} H^{0}\left(M, L^{N}\right)$. For notation and background we refer to [GH, SZ2. We normalize the volume form by

$$
d \tilde{V}:=\left(\frac{\omega(z)}{\pi}\right)^{m}, \quad \int_{M}\left(\frac{\omega}{\pi}\right)^{m}=1 .
$$

We denote the zero set of a full system $\left\{s_{1}^{N}, \ldots, s_{m}^{N}\right\}$ of $m$ polynomials (or sections) in dimension $m$ by

$$
Z_{s_{1}^{N}, \cdots, s_{m}^{N}}=\left\{\left(z^{1}, \ldots, z^{m}\right) \in M: s_{j}^{N}\left(z^{1}, \ldots, z^{m}\right)=0, \forall j=1, \ldots, m\right\} .
$$


The Riesz energy (11) of a configuration of zeros is thus given by

$$
\mathcal{E}_{s}\left(Z_{s_{1}^{N}, \cdots, s_{m}^{N}}\right)=\sum_{\substack{z^{i} \neq z^{j} \\ z^{i}, z^{j} \in Z_{s_{1}^{N}, \cdots, s_{m}^{N}}}} G_{s}\left(z^{i}, z^{j}\right),
$$

where $G_{s}$ is defined in (21).

As in SZ1, SZ2, BSZ1 and elsewhere, we endow the spaces $H^{0}\left(M, L^{N}\right)$ with Gaussian probability measures. They are induced by the inner product defined as follows: Let $h^{N}$ on $L^{N}$ denote the $N$ th power of the Hermitian metric $h$ on $L$, and define

$$
\left\langle s_{1}, s_{2}\right\rangle=\int_{M} h^{N}\left(s_{1}, s_{2}\right) \frac{1}{m !} \omega^{m}, \quad s_{1}, s_{2} \in H^{0}\left(M, L^{N}\right) .
$$

The Hermitian Gaussian measure on $H^{0}\left(M, L^{N}\right)$ is the complex Gaussian probability measure $\mu_{h}^{N}$ induced on $H^{0}\left(M, L^{N}\right)$ by the inner product (11):

$$
d \mu_{h}^{N}(s)=\frac{1}{\pi^{m}} e^{-|c|^{2}} d c, \quad s=\sum_{j=1}^{d_{N}} c_{j} S_{j}^{N},
$$

where $\left\{S_{1}^{N}, \ldots, S_{d_{N}}^{N}\right\}$ is an orthonormal basis for $H^{0}\left(M, L^{N}\right)$.

The Gaussian measure $d \mu_{h}^{N}$ induces product measures on $\left(H^{0}\left(M, L^{N}\right)\right)^{k}=$ $H^{0}\left(M, L^{N}\right) \times \cdots \times H^{0}\left(M, L^{N}\right)(k$ times $)$. For simplicity of notation we continue to denote them by $d \mu_{h}^{N}$, leaving it to the context to make it clear how may factors of $H^{0}\left(M, L^{N}\right)$ are involved. The product measure corresponds to choosing $k \leq m$ independent random sections, hence a Gaussian random system. We define $E_{\mu_{h}^{N}} \mathcal{E}_{s}$ to be the expected (average) value of the energy of the zeros of Gaussian random sections chosen from the ensemble $\left(H^{0}\left(M, L^{N}\right), \mu_{h}^{N}\right)$. In the discrete case,

$$
E_{\mu_{h}^{N}} \mathcal{E}_{s}=\int_{\left(H^{0}\left(M, L^{N}\right)\right)^{m}} \mathcal{E}_{s}\left(Z_{s_{1}^{N}, \cdots, s_{m}^{N}}\right) d \mu_{h}^{N}(s)=\int_{\left(\mathbb{C}^{\left.d_{N}\right)^{m}}\right.} \mathcal{E}_{s}\left(Z_{s_{1}^{N}, \cdots, s_{m}^{N}}\right) \frac{e^{-|c|^{2}}}{\pi^{d_{N}}} d c .
$$

Before stating our results in the discrete case, we recall the results of $[\mathrm{Z}, \mathrm{ABS}]$ when $m=1$. In the special case of the standard metric on $S^{2}$ and with the logarithmic Riesz energy with respect to the chordal distance, it is proved in [Z, ABS that

$$
E_{\mu_{h_{F S} N}} \mathcal{E}_{0}=\left\{\begin{array}{cl}
\frac{N^{2}}{4}-\frac{1}{4} N \log N-\frac{N}{4}, & \text { radius }=\frac{1}{2}, \\
-\frac{1}{4} \log \left(\frac{4}{e}\right) N^{2}-\frac{1}{4} N \log N+\frac{1}{4} \log \left(\frac{4}{e}\right) N, & \text { radius }=1 .
\end{array}\right.
$$

In our notation, $S^{2}$ is the Kähler manifold $\left(\mathbb{C P}^{1}, \omega_{F S}\right)$, where $\omega_{F S}$ (or $h_{F S}$ ) is the Fubini-Study metric, and the holomorphic line bundle $L$ is the hyperplane line bundle $\mathcal{O}(1)$; the area of $\left(\mathbb{C P}^{1}, \omega_{F S}\right)$ equals 1 , hence is the round $S^{2}$ of radius $\frac{1}{2}$. The expected energy of $N$ randomly chosen points (i.e. from the binomial point process) equals $-\frac{1}{4} \log \left(\frac{4}{e}\right) N^{2}+\frac{1}{4} \log \left(\frac{4}{e}\right) N$. Thus, the $-N \log N$ term reflects the lower energy of well-separated points.

To be precise, Q. Zhong [Z] obtained general asymptotic results for any Kähler metric on any Riemann surface, where the energy was defined in terms of the Green's function $G_{0}(z, w)$, i.e. the kernel of $\Delta_{g}^{-1}$ on the orthogonal complement of 
the constant functions. It has a well-known expansion

$$
G_{0}(z, w)=-\frac{1}{2} \log r_{g}(z, w)+F_{g}(z, w)
$$

around the diagonal, where $F_{g}$ is continuous (it is known as the Robin function). It has the long-range property that $\int_{M} G_{0}(z, w) \omega(z)=0$. In the case of the Green's energy, Zhong proved that

$$
E_{\mu_{h}^{N}} \mathcal{E}_{0}^{N}=-\frac{1}{4} N \log N-\frac{N}{4}-N \int_{M} F_{g}(z, z) \omega+o(N),
$$

where $\int_{M} F_{g}(z, z) \omega$ is the Robin constant. The $N^{2}$ term vanishes since the integration of the Green's function is 0 . In the special case of the standard metric on $S^{2}$, Armentano-Beltran-Shub ABS] later showed that the expected energy is exactly given by the formulae in (14) without the $o(N)$ term. The general result of $[\mathrm{Z}$ only gives the result with the $o(N)$ remainder estimate above.

The asymptotic results of $[\mathrm{Z}, \mathrm{ABS}]$ show that the expected energy of zero point configurations is asymptotically the same as the minimum energy of any configuration up to order $N$ (it appears to remain unknown what the coefficient of the $N$ term is for the minimal energy). See also [ZZe for a correction and addendum to the calculations in [Z]. The expected Green's kernel energy is infinite in complex dimension $>2$, so we do not emphasize it here.

1.3. Main results in the discrete case. In this article, we consider random Riesz $s$-energies for zeros of polynomial systems and their analogues on a general compact Kähler manifold of any dimension $m$ and for a certain range of $s$. As discussed above, we consider $k \leq m$ independent Gaussian random holomorphic sections of $L^{N} \rightarrow M$ where $(L, h) \rightarrow M$ is a positive Hermitian line bundle. The Gaussian measure (12) is the same as in [SZ1, SZ2] (see \$2.2 for background). As above, our Gaussian random systems of $k \leq m$ sections are random elements of the product space $\left(H^{0}\left(M, L^{N}\right)\right)^{k}$ equipped with product Gaussian measure. In the discrete case we obtain the following asymptotic expansion for the average Riesz energy defined in (2). The dependence of the results on $s$ are illustrated by the graphics in 4

Theorem 1. Let $(M, \omega)$ be an m-dimensional compact Kähler manifold and let $(L, h) \rightarrow(M, \omega)$ be a positive Hermitian holomorphic line bundle. Then the expected Riesz energies defined in (13) admit the following asymptotics:

- If $0<s<\min \{2 m, 4\}$,

$$
\begin{aligned}
E_{\mu_{h}^{N}} \mathcal{E}_{s}= & a_{1}(h, s) N^{2 m}+\sum_{j=2}^{p-1} a_{j}(h, s) N^{2 m-j} \\
& +c_{m}(s) N^{m+\frac{s}{2}}+O\left(N^{m+\frac{s-1}{2}}(\log N)^{m-\frac{s}{2}}\right),
\end{aligned}
$$

where $p=\left[m-\frac{s}{2}\right]+1$, where $a_{j}(h, s)$ are the coefficients in Lemma 1, and where

$$
c_{m}(s)=2 m \int_{0}^{\infty}\left[\kappa_{m m}(r)-1\right] r^{2 m-1-s} d r .
$$


- In the case of logarithmic energy when $s=0$, we have

$$
\begin{aligned}
E_{\mu_{h}^{N}} \mathcal{E}_{0} & =a_{1}(h, 0) N^{2 m}+a_{2}(h, 0) N^{2 m-2}+\cdots+\left[a_{m}(h, 0)-c_{m}\right] N^{m} \\
& -N^{m} \log \sqrt{N}+O\left(N^{m-\frac{1}{2}}(\log N)^{m+1}\right),
\end{aligned}
$$

where

$$
c_{m}=2 m \int_{0}^{\infty} \log r\left[\kappa_{m m}(r)-1\right] r^{2 m-1} d r .
$$

Above, the coefficient $c_{m}(s)$ involves the scaling limit $\kappa_{m m}(r)$ of the pair correlation function of zeros in the discrete case in dimension $m$. As reviewed in 2.4 , it has the small distance asymptotics,

$$
\kappa_{m m}(r)=\frac{m+1}{4} r^{4-2 m}+O_{m}\left(r^{8-2 m}\right), \quad \text { as } r \rightarrow 0 .
$$

Hence the integral defining the coefficient $c_{m}(s)$ only converges if $s \leq 4$. It is independent of the Hermitian metric $h$.

The coefficients $a_{j}(h, s)$ are integrals of $G_{s}(z, w)$ against curvature invariants of $h$ and can be explicitly computed by the algorithm in Lemma 1. In particular, the first two are given by

$$
\begin{aligned}
& a_{1}(h, s)=\int_{M \times M} G_{s}(z, w) d \tilde{V}(z) d \tilde{V}(w), \\
& a_{2}(h, s)=-2 \int_{M \times M} G_{s}(z, w) \Delta_{\omega} S(z) d \tilde{V}(z) d \tilde{V}(w),
\end{aligned}
$$

where $\Delta_{\omega}$ is the complex Laplace operator with respect to the Kähler form $\omega$ and $S$ is the scalar curvature of $\omega$.

We can give a more explicit evaluation in the special case of $\left(\mathbb{C P}^{m}, \omega_{F S}\right)$ and $(L, h)=\left(\mathcal{O}(1), h_{F S}\right)$. As is well known, in a suitable frame, we can identify $H^{0}\left(\mathbb{C P}^{m}, \mathcal{O}(N)\right)$ with the space of polynomials of $m$ variables of degree $N$. Namely, one homogenizes the polynomials $p_{N}$ to be homogeneous polynomials $\hat{p}_{N}$ of degree $N$ on $\mathbb{C}^{m+1}$ and then restricts $\hat{p}_{N}$ to the unit sphere $S^{2 m+1} \subset \mathbb{C}^{m+1}$. They transform by $e^{i N \theta}$ under the $S^{1}$ action for the Hopf principal bundle $S^{2 m+1} \rightarrow \mathbb{C P}^{m}$, and in a well-known way descend to $\mathbb{C P}^{m}$ as holomorphic sections of $\mathcal{O}(N)$. We refer to [GH] (pp. 165-166) or to [SZ1, SZ2 for more details.

Corollary 1. For the space $H^{0}\left(\mathbb{C P}^{m}, \mathcal{O}(N)\right)$ of polynomials of degree $N$ equipped with the Gaussian Hermitian measure induced by $h_{F S}$, the Riesz energies of zeros of $m$ independent random polynomials are given by

- If $0<s<\min \{2 m, 4\}$,

$$
E_{\mu_{h}^{N}} \mathcal{E}_{s}=a_{1}\left(h_{F S}, s\right) N^{2 m}+c_{m}(s) N^{m+\frac{s}{2}}+O\left(N^{m+\frac{s-1}{2}}(\log N)^{m-\frac{s}{2}}\right) .
$$

- For the case of logarithmic energy, we have

$$
E_{\mu_{h}^{N}} \mathcal{E}_{0}=a_{1}\left(h_{F S}, 0\right) N^{2 m}-N^{m} \log \sqrt{N}-c_{m} N^{m}+O\left(N^{m-\frac{1}{2}}(\log N)^{m+1}\right) .
$$

Thus, all of the terms $a_{j}\left(h_{F S}, s\right)$ vanish for $j \geq 2$. The proof follows directly from the proof of Lemma 1 and is based on the fact that the Fubini-Study metric is balanced, i.e. the Szegö kernel is constant on the diagonal. The third coefficient of the logarithmic energy asymptotics differs from that of (16) because Zhong used $\frac{1}{2} \log [z, w]$ for the logarithmic kernel. 
The asymptotics reflect the local behavior (repulsion/attraction) of the zeropoint configurations and the long-range order (uniform distribution) as follows:

- The leading coefficient $a_{1}(h, s)$ is the "Poisson term", i.e. the scaling limit of the expected energy of a binomial point process where one chooses $N^{m}$ points at random from $M$, i.e. a configuration at random from $M^{\left(N^{m}\right)}=$ $\underbrace{M \times \cdots \times M}_{N^{m}} / S_{N^{m}}$ (the $N^{m}$-fold symmetric product) with respect to $d \tilde{V}$. See $\$ 2.5$ for further discussion.

- The coefficients $a_{j}(h, s)$ are global lower order corrections due to the nonconstant curvature of the Kähler metric.

- The terms $c_{m}(s) N^{m+\frac{s}{2}}$ and $-N^{m} \log \sqrt{N}-c_{m} N^{m}$ reflect the local behavior (i.e. separation, repulsion or clumping) of the zeros. They are derived from the integration of Riesz functions against the current of two point correlations near the diagonal of $M \times M$, i.e. they arise from term I localized in a $\frac{\log N}{\sqrt{N}}$ neighborhood of the diagonal in 3.1 . The separation of the zeros depends on the dimension $m$.

- The overall asymptotics reflects a competition between the global and local behavior of zeros. The larger $s$ is, the more significant the separation of zeros. When $s>2$, the local behavior of zero-point configurations dominates the global behavior; when $s<2$, the global behavior dominates the local one.

The asymptotic average energy of zero point configurations may be compared with the minimum energy (7), keeping in mind that the expected number of points in the configuration of zeros is $N^{m}$. Of course, $S^{n}$ is not a Kähler manifold for $n \geq 3$, so no exact comparison can be made between the minimum and average energies. The closest Kähler analogue of a round sphere $S^{2 m}$ is $\mathbb{C P}^{m}$ with its Fubini-Study metric of constant positive curvature. For Riesz energies with $0<s<\min \{2 m, 4\}$ the results of Corollary 1 may be compared with the result for the standard $S^{2 m}$ where

$$
\frac{1}{2} V_{2 m}(s) N^{2 m}-C_{3}(s) N^{m+s / 2} \leq \mathcal{E}_{s}\left(N^{m}\right) \leq \frac{1}{2} V_{2 m}(s) N^{2 m}-C_{4}(s) N^{m+s / 2} .
$$

The exponent $s / 2$ arises because we have $N^{m}$ points in real dimension $2 m$. The leading coefficients of the minimum and average energies are both given by the Poissonian term and may be considered as the same. Hence the difference between minimum energy and mean energy of zero point configurations is first detected in the subleading terms of order $N^{m+\frac{s}{2}}$, which as discussed above is due to the separation between points. The coefficient $C_{j}(s)$ is not known explicitly, but it is significant to compare the signs of $C_{j}(s)$ and $c(s)$. It is known that $C_{j}(s)>0$, so that the subleading term of the minimum energy is negative. Intuitively, we expect that $c_{m}(s)>0$ for $s$ near 4 when $m \geq 3$ due to the clumping of the zeros as compared to the repulsion that must take place in an optimal configuration. The exact formula for $\kappa_{m m}(r)$ is given in [BSZ3] and recalled in (46). It is very complicated and represents the pair correlation function for zeros of random entire functions in the Bargmann-Fock model, i.e. entire analytic functions on $\mathbb{C}$ which are in $L^{2}\left(\mathbb{C}, e^{-|z|^{2}}\right) . \kappa_{m m}(r)$ blows up to $+\infty$ for $r \rightarrow 0$ if $m \geq 3$, supporting the intuition that $c_{m}(s)$ should be positive. But it also has negative values in a certain interval, which represents a kind of repulsion relative to the Poisson spatial process for intermediate values of $r$, and this complicates the discussion. 
However, we can justify the intuition for $s$ near 4 :

Proposition 1. For any $m \geq 3$, there exists $s_{m} \in[0,4)$ so that for $s \geq s_{m}$,

$$
c_{m}(s)=2 m \int_{0}^{\infty}\left[\kappa_{m m}(r)-1\right] r^{2 m-1-s} d r>0 .
$$

We do not try to find a good value of $s_{m}$, which probably requires a computer assisted calculation, but are content with a simple argument that nevertheless clarifies the structure of $\kappa_{m m}(r)$.

1.4. The higher codimension case. The methods extend readily to systems of $k \leq m$ polynomials (or sections) whose zero set is a complex submanifold of codimension $k$, so we also include this case. If $A \subset M$ is a complex subvariety of codimension $k \leq m-1$, the Riesz functions have no point mass on the diagonal, and we define the continuous Riesz energies as

$$
\mathcal{E}_{s}(A)=\iint_{A \times A} G_{s}(z, w) d V_{k}(z) d V_{k}(w),
$$

where $d V_{k}=\frac{\omega^{m-k}}{(m-k) !}$ is the volume form on $A$.

The zero locus $Z_{s_{1}^{N}, \cdots, s_{k}^{N}}$ of $k$ holomorphic random sections is an analytic subvariety of codimension $k$ where $k<m$. We define

$$
E_{\mu_{h}^{N}} \mathcal{E}_{s}=\int_{\left(H^{0}\left(M, L^{N}\right)\right)^{k}} \mathcal{E}_{s}\left(Z_{s_{1}^{N}, \cdots, s_{k}^{N}}\right) d \mu_{h}^{N}(s)=\int_{\left(\mathbb{C}^{d_{N}}\right)^{k}} \mathcal{E}_{s}\left(Z_{s_{1}^{N}, \cdots, s_{k}^{N}}\right) \frac{e^{-|c|^{2}}}{\pi^{d_{N}}} d c,
$$

where

$$
\mathcal{E}_{s}\left(Z_{s_{1}^{N}, \cdots, s_{k}^{N}}\right)=\int_{Z_{s_{1}^{N}, \ldots, s_{k}^{N}} \times Z_{s_{1}^{N}, \ldots, s_{k}^{N}}} G_{s}(z, w) d V_{k}(z) d V_{k}(w) .
$$

We then have,

Theorem 2. With the same assumptions as in Theorem 1,

- If $0<s<2(m-k)$, we have

$$
\begin{aligned}
E_{\mu_{h}^{N}} \mathcal{E}_{s}= & \left(\frac{\pi^{m-k}}{(m-k) !}\right)^{2}\left[a_{1}(h, s) N^{2 k}+\sum_{j=2}^{p-1} w_{j}(k) a_{j}(h, s) N^{2 k-j}\right] \\
& +d_{m}(k, s) N^{2 k-m+\frac{s}{2}}+O\left(N^{2 k-m+\frac{s-1}{2}}(\log N)^{m-\frac{s}{2}}\right),
\end{aligned}
$$

where $p=\left[m-\frac{s}{2}\right]+1$ and $d_{m}(k, s)$ is the integral defined in (77).

- If $s=0$, we have

$$
\begin{aligned}
E_{\mu_{h}^{N}} \mathcal{E}_{0}= & \left(\frac{\pi^{m-k}}{(m-k) !}\right)^{2}\left[a_{1}(h, 0) N^{2 k}+w_{2}(k) a_{2}(h, 0) N^{2 k-2}\right. \\
& \left.+\cdots+w_{m}(k) a_{m}(h, 0) N^{2 k-m}\right] \\
- & d_{m}(k) N^{2 k-m}+e(k) N^{2 k-m} \log \sqrt{N}+O\left(N^{2 k-m-\frac{1}{2}}(\log N)^{m+1}\right),
\end{aligned}
$$

where $d(k)$ and $e(k)$ are constants depending on $k$,

where $a_{j}(h, s)$ is defined in Theorem 1 and $w_{j}(k)$ is the weight; in particular, $w_{2}(k)=\frac{k}{m}$. 
Remark 1. Note that conditions of $s$ in the two theorems are different. This is because the behavior of the pair correlation functions of zeros near the origin are different when $k \neq m$ and $k=m$, respectively.

1.5. Systems with varying degrees. We have chosen to work with $k$ independent Gaussian random sections of $H^{0}\left(M, L^{N}\right)$, i.e. to all be of degree $N c_{1}(L)$, but it is possible to generalize the results to cases where the degrees are chosen differently for the different sections. Instead of $\left(H^{0}\left(M, L^{N}\right)^{k}\right.$ we would work with $\prod_{j=1}^{k} H^{0}\left(M, L^{N_{j}}\right)$, where the degrees $N_{j} \rightarrow \infty$. The formula (32) would generalize to a product where the degrees would vary with the section. One would then obtain joint asymptotics in the vector of degrees $\left(N_{1}, \ldots, N_{k}\right)$. The simplest modification would be to choose the degrees to have the form $N\left(\ell_{1}, \ldots, \ell_{k}\right)$ with a lattice point $\left(\ell_{1}, \ldots, \ell_{k}\right) \in \mathbf{Z}^{k}$. For the sake of simplicity we only consider the case $\left(\ell_{1}, \ldots, \ell_{k}\right)=(1,1, \ldots, 1)$.

1.6. Fekete points. Although we do not have any results on it, we should point out that the separation and equidistribution of points in dimensions $m>1$ are often measured by the energies of Leja and Zaharjuta,

$$
\log \Delta_{N}\left(z_{1}, \ldots, z_{d_{N}}\right)=\log \operatorname{det}\left(B_{N}\left(z_{i}, z_{j}\right)\right),
$$

where $B_{N}$ is the Bergman kernel for degree $N$ polynomials (the kernel of the orthogonal projection onto the degree $N$ polynomials). Here, $d_{N}$ is the dimension of the space of polynomials of degree $N$. In dimension one, this is a Vandermonde type determinant whose logarithm is closely related to the Green's energy of the configuration. But in higher dimensions, the Green's energy and $\log \operatorname{det}\left(B_{N}\left(z_{i}, z_{j}\right)\right)$ are no longer known to be closely related and probably are not. Moreover, one is forced to choose different numbers of points for the two different energies: for (23) one chooses $d_{N}$ points, but in the Green's energy problem in the point case the number of zeros of the system is $c_{1}\left(L^{N}\right)^{m}>d_{N}$. We refer to BBWN for recent results on Fekete points in higher dimensions.

\section{BACKGROUND}

In this section, we recall some definitions in Kähler geometry GH, Ze and some properties about zeros of random holomorphic sections of positive holomorphic line bundles BSZ1, BSZ2, BSZ3.

2.1. Kähler geometry. Let $(M, \omega)$ be an $m$-dimensional polarized compact Kähler manifold. Then there exists a polarization of $(M, \omega)$; i.e., there exists a positive holomorphic line bundle $(L, h)$ with smooth Hermitian metric $h$ such that the curvature form

$$
\Theta_{h}=-\partial \bar{\partial} \log \left\|e_{L}\right\|_{h}^{2}
$$

satisfies the condition

$$
\omega=\frac{\sqrt{-1}}{2} \Theta_{h} .
$$

Here, $e_{L}$ is a local non-vanishing holomorphic section of $L$ over an open set $U \subset M$, and $\left\|e_{L}\right\|_{h}=h\left(e_{L}, e_{L}\right)^{1 / 2}$ is the $h$-norm of $e_{L}[\mathrm{GH}$.

We denote by $H^{0}\left(M, L^{N}\right)$ the space of global holomorphic sections of $L^{N}=$ $L \otimes \cdots \otimes L$. The metric $h$ induces Hermitian metrics $h^{N}$ on $L^{N}$ given by $\left\|s^{\otimes N}\right\|_{h^{N}}=$ $\|s\|_{h}^{N}$. We give $H^{0}\left(M, L^{N}\right)$ the Hermitian inner product (11). 
We define the Bergman kernel as the orthogonal projection from the $L^{2}$ integral sections to the holomorphic sections:

$$
\Pi_{N}(z, w): L^{2}\left(M, L^{N}\right) \rightarrow H^{0}\left(M, L^{N}\right)
$$

with respect to the inner product $\langle\cdot, \cdot\rangle_{h^{N}}$.

Denote $\left\{S_{1}^{N}, \cdots, S_{d_{N}}^{N}\right\}$ as the orthonormal basis of $H^{0}\left(M, L^{N}\right)$ with respect to the inner product $\langle\cdot, \cdot\rangle_{h^{N}}$, where $d_{N}=\operatorname{dim} H^{0}\left(M, L^{N}\right)$. Then along the diagonal, we have the following complete asymptotics $[\mathrm{L}, \mathrm{Ze}$ :

$$
\Pi_{N}(z, z)=\sum_{j=1}^{d_{N}}\left\|S_{j}^{N}\right\|_{h^{N}}^{2}=N^{m}+a_{1}(z) N^{m-1}+a_{2}(z) N^{m-1} \cdots,
$$

where each coefficient $a_{j}(z)$ is a polynomial of the curvature and its covariant derivatives. In particular, $a_{1}(z)$ is the scalar curvature of $\omega$.

Example. In the case of $\left(\mathbb{C P}^{m}, \omega_{F S}\right)$ with the line bundle $\left(\mathcal{O}(N), h_{F S}\right)$ which is the $N$ th power of the hyperplane line bundle $\mathcal{O}(1)$, the Bergman kernel is a constant along the diagonal BSZ2],

$$
\Pi_{N}(z, z)=\frac{(N+m) !}{N !}
$$

2.2. Gaussian random systems. We now equip the vector space $H^{0}\left(M, L^{N}\right)$ with the complex Gaussian probability measure (12) induced by the inner product $\langle\cdot, \cdot\rangle_{h^{N}}$. We express any holomorphic section as a series

$$
s=\sum_{j=1}^{d_{N}} c_{j} S_{j}^{N}
$$

relative to an orthornormal basis $\left\{S_{j}^{N}\right\}$ for $\langle\cdot, \cdot\rangle_{h^{N}}$. The corressponding Gaussian measured is characterized by the property that the $2 d_{N}$ real variables $\Re c_{j}$, $\Im c_{j}$ $\left(j=1, \ldots, d_{N}\right)$ are independent random variables with mean 0 and variance $\frac{1}{2}$; i.e.,

$$
E c_{j}=0, \quad E c_{j} c_{k}=0, \quad E c_{j} \bar{c}_{k}=\delta_{j k},
$$

where $E$ denotes the expectation.

We then endow the product space $\left(H^{0}\left(M, L^{N}\right)\right)^{k}(k \leq m)$ with the product measure, which we continue to denote by $d \mu_{h}^{N}$.

2.3. Zeros of random sections. For $k$ holomorphic sections $s_{1}^{N}(z), \cdots, s_{k}^{N}(z) \in$ $\left(\left(H^{0}\left(M, L^{N}\right)\right)^{k}, d \mu_{h}^{N}\right)$ with $k \leq m$, we denote it by

$$
Z_{s_{1}^{N}, \cdots, s_{k}^{N}}:=\left\{z \in M: s_{1}^{N}(z)=\cdots=s_{k}^{N}(z)=0\right\} .
$$

It is a subvariety of codimension $k$. The current of integration over the zero locus is defined by

$$
\left(Z_{s_{1}^{N}, \cdots, s_{k}^{N}}, \phi\right)=\int_{Z_{s_{1}^{N}, \ldots, s_{k}^{N}}} \phi(z), \phi \in \mathcal{D}^{m-k, m-k}(M),
$$

which we also write as

$$
\left(Z_{s_{1}^{N}, \cdots, s_{m}^{N}}, \phi\right)=\int_{M} Z_{s_{1}^{N}, \cdots, s_{k}^{N}} \wedge \phi(z), \phi \in \mathcal{D}^{m-k, m-k}(M) .
$$


For the special point case of $k=m$,

$$
\left(Z_{s_{1}^{N}, \cdots, s_{m}^{N}}, \phi\right)=\sum_{s_{1}^{N}(z)=\cdots=s_{m}^{N}(z)=0} \phi(z), \phi \in C(M) .
$$

We denote by $Z_{s^{N}}$ the current of integration over the zero locus of a single section. By the Poincaré-Lelong formula, we have (as in SZ1] )

$$
E\left(Z_{s^{N}}\right)=\frac{\sqrt{-1}}{2 \pi} \partial \bar{\partial} \log \Pi_{N}+\frac{N}{\pi} \omega .
$$

It follows by (25) that

$$
E\left(\frac{1}{N} Z_{s^{N}}\right) \rightarrow \frac{\omega}{\pi} \text { as } N \rightarrow \infty
$$

Define

$$
E_{k m}^{N}:=E\left(Z_{s_{1}^{N}, \cdots, s_{k}^{N}}\right), \quad k \leq m
$$

as the expectation of a zero locus of $k$ independent random holomorophic sections of $L^{N}$. Then we have [SZ1,

$$
E_{k m}^{N}=\left(E\left(Z_{s^{N}}\right)\right)^{k}=\left(\frac{i}{2 \pi} \partial \bar{\partial} \log \Pi_{N}(z, z)+\frac{N}{\pi} \omega\right)^{k} .
$$

By 25) we get

$$
E\left(Z_{s_{1}^{N}, \cdots, s_{k}^{N}}\right)=\frac{N^{k}}{\pi^{k}} \omega^{k}+O\left(N^{m-1}\right)
$$

for $N$ large enough. As a direct consequence, we know the expected number of discrete zeros is $r_{N}=\left(c_{1}\left(L^{N}\right)\right)^{m}$ when $k=m$.

Example. In the case $\mathcal{O}(N) \rightarrow \mathbb{C P}^{m}$ equipped with the Fubini-Study metric, $H^{0}\left(\mathbb{C P}^{m}, \mathcal{O}(N)\right)$ is identified with the space of polynomials of degree $N$. We equip $H^{0}\left(\mathbb{C P}^{m}, \mathcal{O}(N)\right)$ with a complex Gaussian ensemble induced by the Fubini-Study metric. Since the Bergman kernel is constant (26), by the Poincaré-Lelong formula (32), the distribution of zeros of Gaussian random polynomials $p^{N}$ satisfies the exact formula,

$$
E\left(\frac{1}{N} Z_{p^{N}}\right)=\frac{\omega_{F S}}{\pi},
$$

as $N$ large enough. Also, the expected number of zeros is $N^{m}$.

2.4. The pair correlation current. The pair correlation current is defined by

$$
K_{k m}^{N}(z, w):=E\left(Z_{s_{1}^{N}, \cdots, s_{k}^{N}}(z) \otimes Z_{s_{1}^{N}, \cdots, s_{k}^{N}}(w)\right) \in \mathcal{D}^{2 k, 2 k}(M \times M)
$$

in the sense that for any test form $\phi_{1}(z) \otimes \phi_{2}(w) \in \mathcal{D}^{m-k, m-k}(M) \otimes \mathcal{D}^{m-k, m-k}(M)$, we have the pairing

$$
\left(K_{k m}^{N}(z, w), \phi_{1}(z) \otimes \phi_{2}(w)\right)=E\left[\left(Z_{s_{1}^{N}, \cdots, s_{k}^{N}}, \phi_{1}\right)\left(Z_{s_{1}^{N}, \cdots, s_{k}^{N}}, \phi_{2}\right)\right] .
$$

In the special case when $k=m$, the correlation measures take the form

$$
K_{m m}^{N}(z, w)=[\triangle] \wedge\left(E_{m m}^{N}(z) \otimes 1\right)+\kappa^{N}(z, w) \omega_{z}^{m} \otimes \omega_{w}^{m},
$$

where $[\triangle]$ denotes the current of the integration along the diagonal, $\kappa^{N} \in C^{\infty}(M \times$ $M)$ and $E_{m m}^{N}$ is the expectation $E\left(Z_{s_{1}^{N}, \cdots, s_{m}^{N}}\right)$. 
The correlation currents of two points $K_{k m}^{N}$ are smooth forms away from the diagonal in $M \times M$ and have no mass on the diagonal for $k<m$. In the case of $k=m, K_{m m}^{N}$ contains a delta-function along the diagonal [SZ1].

If $1 \leq k \leq m-1$, the correlation current for $K_{k m}^{N}$ is an $L^{1}$ current on $M \times M$ given by

$$
K_{k m}^{N}=\left(-\partial_{1} \bar{\partial}_{1} \partial_{2} \bar{\partial}_{2} Q_{N}+E Z_{s^{N}} \otimes E Z_{s^{N}}\right)^{k},
$$

where $Q_{N}$ is the pluri-bipotential defined in [SZ1].

In the case of $k=m$,

$$
K_{m m}^{N}=\left.\left(-\partial_{1} \bar{\partial}_{1} \partial_{2} \bar{\partial}_{2} Q_{N}+E Z_{s^{N}} \otimes E Z_{s^{N}}\right)^{m}\right|_{M \times M \backslash \triangle}+\operatorname{diag}_{*}\left(E Z_{s^{N}}\right)^{m},
$$

where $\triangle$ is the diagonal in $M \times M$ and diag : $M \rightarrow M \times M$ is the diagonal map $\operatorname{diag}(z)=(z, z)$.

Furthermore, the pair correlation currents has the off-diagonal asymptotics [SZ1,

$$
K_{k m}^{N}=\left(E Z_{s^{N}} \otimes E Z_{s^{N}}\right)^{k}+O\left(N^{-q}\right), \text { for } r_{g}(z, w) \geq b \sqrt{\frac{\log N}{N}},
$$

where $b>\sqrt{q+2 k+3} \geq 3$.

2.4.1. Universal rescaling property. We now rescale the correlation functions around a point $z_{0} \in M$ in normal coordinates by a factor of $\sqrt{N}$. In BSZ2, the authors prove that the pair correlation currents have the universal rescaling asymptotics,

$$
K_{k m}^{N}\left(\frac{z}{\sqrt{N}}, \frac{w}{\sqrt{N}}\right)=K_{k m}^{\infty}(z, w)\left(\frac{i}{2 \pi} \partial \bar{\partial}|z|^{2}\right)^{k} \wedge\left(\frac{i}{2 \pi} \partial \bar{\partial}|w|^{2}\right)^{k}+O\left(\frac{1}{\sqrt{N}}\right),
$$

where the error term is $\frac{1}{\sqrt{N}}$ times a smooth volume form. The universal rescaling property is independent of the manifold $M$ and the line bundle $L ; K_{k m}^{\infty}$ depends only on the dimension $m$ of the manifold and the codimension $k$ of the zero set. Furthermore, $K_{k m}^{\infty}(z, w)$ is a function depending on the distance $r_{g}(z, w)$, and thus we rewrite [BSZ3]:

$$
K_{k m}^{\infty}(z, w)=\kappa_{k m}(r), \quad r=|z-w|, \quad 1 \leq k \leq m .
$$

The universal scaling limit $\kappa_{k m}(r)$ is explicitly computed in [BSZ2, BSZ3]. In the case when $k=m$, one has the rather complicated formula $\left(v=e^{-r^{2}}\right)$

$$
\kappa_{m m}(r)=\frac{m\left(1-v^{m+1}\right)(1-v)+r^{2}(2 m+2)\left(v^{m+1}-v\right)+r^{4}\left[v^{m+1}+v^{m}+(\{m+1\} v+1)\left(v^{m}-v\right) /(v-1)\right]}{m(1-v)^{m+2}} .
$$

For small values of $r$, we have

$$
\kappa_{m m}(r)=\frac{m+1}{4} r^{4-2 m}+O\left(r^{8-2 m}\right), \quad \text { as } r \rightarrow 0 .
$$

As mentioned above, the $\kappa_{11}(r) \sim r^{2}$ as $r \rightarrow 0$, exhibiting repulsion, while $\kappa_{22}(r) \rightarrow$ $\frac{3}{4}$ when $m=2$. For $m \geq 3, \kappa_{m m}(r)$ blows up as $r \rightarrow 0$.

In the higher codimension case $k \neq m$,

$$
\kappa_{k m}(r) \sim r^{-2 k}, \text { as } r \rightarrow 0 .
$$

In both cases,

$$
\kappa_{k m}(r)=1+O\left(r^{4} e^{-r^{2}}\right), \quad \text { as } r \rightarrow \infty,
$$


for any $1 \leq k \leq m$. Thus, the correlations are very short range on the $\frac{1}{\sqrt{N}}$ length scale. The constant 1 would be the correlation function for the Poisson spatial process (i.e. independent points chosen at random from the normalized volume form). Below are computer graphics of $\kappa_{m m}(r)$ in the cases $m=2,3,4$.
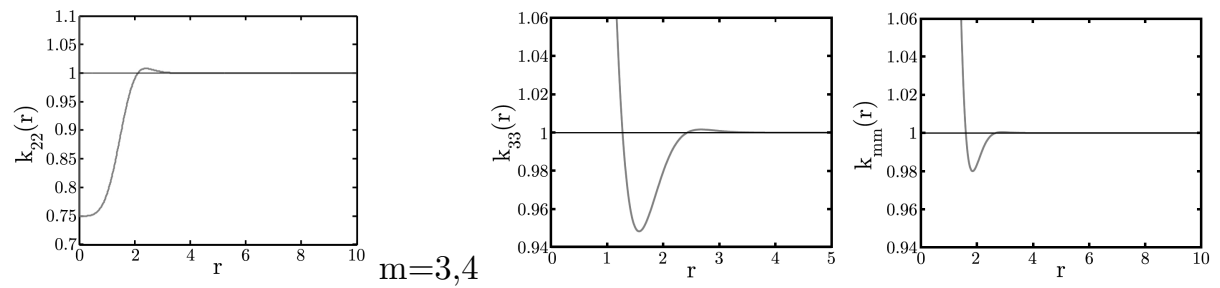

From the computer graphic, one sees that $\kappa_{33}(r)-1$ and $\kappa_{44}-1$ each have two zeros, both less than 4 , and each is positive elsewhere. In Proposition 1 we show that $k_{m m}(r)-1>0$ when $m \geq 3$ for $r^{2} \geq 2 m+3$, but almost certainly it becomes positive at much smaller values of $r$.

2.5. Configuration spaces, empirical measures and random points. Generic configurations of zeros $\zeta=\left\{\zeta^{1}, \ldots, \zeta^{r_{N}}\right\}$ in the discrete (point) case are points in the punctured product

$$
M_{r_{N}}=\{\left(\zeta^{1}, \ldots, \zeta^{r_{N}}\right) \in \underbrace{M \times \cdots \times M}_{r_{N}}: \zeta^{p} \neq \zeta^{q} \text { for } p \neq q\} / S_{r_{N}},
$$

where $r_{N}=\left(c_{1}\left(L^{N}\right)\right)^{m}$ is the number of zeros of the system and $S_{r_{N}}$ is the symmetric group on $r_{N}$ letters. As in the case $m=1$, one has a map

$$
\mu_{\zeta}: M_{r_{N}} \rightarrow \mathcal{P}_{M}, \quad \mu_{\zeta}=\frac{1}{r_{N}} \sum \delta_{\zeta_{j}}
$$

from configurations to probability measures on $M$. We also have the map (9)

$$
\left(s_{1}^{N}, \cdots, s_{m}^{N}\right) \in\left(H^{0}\left(M, L^{N}\right)\right)^{m} \rightarrow Z_{s_{1}^{N}, \cdots, s_{m}^{N}} \in M_{r_{N}}
$$

from polynomial systems to their zeros, and the composition gives the empirical measure of zeros map

$$
\left(s_{1}^{N}, \cdots, s_{m}^{N}\right) \rightarrow \mu_{\zeta}
$$

The Gaussian product probability measure on $\left(H^{0}\left(M, L^{N}\right)\right)^{m}$ pushes forward to a probability measure on $M_{r_{N}}$, and the two-point correlation function (38) is the expected value of $\mu_{\zeta} \otimes \mu_{\zeta}$ with respect to this pushforward. Note that the image in $M_{r_{N}}$ of the zeros map (9) has positive codimension growing linearly in $N$, and therefore the induced measure on $M_{r_{N}}$ is very singular.

By comparison, just choosing $r_{N}$ random points on $M$ from the normalized volume form (8) corresponds to product measures on $M_{r_{N}}$, known as the binomial point process. If we let $N \rightarrow \infty$ and rescale as above, we get the Poisson point process on $\mathbb{C}^{m}$ with intensity given by the Euclidean measure. Its correlation function is then the constant function 1 . Hence $\kappa_{m m}-1$ represents the deviation of the zero point correlations from the Poisson case.

The Riesz energies of configurations (11) are functions on $M_{r_{N}} \times M_{r_{N}}$ which blow up on the 'large diagonals' where some pair $\zeta^{i}=\zeta^{j}(i \neq j)$. For polynomial systems, this large diagonal defines the discriminant variety $\mathcal{D}_{m, N}$ of full polynomial 
systems of degree $N$ in $m$ variables which have a double root. Thus $\mathcal{E}_{s}$ defines a simple notion of 'inverse distance to $\mathcal{D}_{m, N}$ '. The large expected value for $\mathcal{E}_{s}$ in dimensions $m \geq 3$ thus show that configurations of zeros are often close to $\mathcal{D}_{m, N}$ by comparison with random points.

\section{Proof of Theorem 1}

Now we compute the Riesz energy of the discrete set $Z_{s_{1}^{N}, \cdots, s_{m}^{N}}$ which is the zero set of $m$ random sections. In the proof, $c$ is a constant, but may be different from line to line. First we need the following lemma.

Lemma 1. We have complete asymptotics,

$$
\begin{aligned}
& \int_{M \times M} G_{s}(z, w) E_{m m}^{N}(z) \wedge E_{m m}^{N}(w) \\
& =a_{1}(h, s) N^{2 m}+a_{2}(h, s) N^{2 m-2}+a_{3}(h, s) N^{2 m-3}+\cdots,
\end{aligned}
$$

where each coefficient $a_{j}(h, s)$ is a constant depending on $s$ and the curvature of $h$; in particular, the first two terms are given in (19).

Proof. From (35), $E_{m m}^{N}(z)$, which is the expectation of discrete zeros of $m$ random sections, is given by the explicit formula

$$
E_{m m}^{N}(z)=\left(\frac{i}{2 \pi} \partial \bar{\partial} \log \Pi_{N}(z, z)+\frac{N}{\pi} \omega(z)\right)^{m} .
$$

First by (25), we have,

$$
\log \Pi_{N}(z, z)=m \log N+a_{1}(z) N^{-1}+\left[a_{2}(z)-\frac{1}{2} a_{1}^{2}(z)\right] N^{-2}+\cdots,
$$

where $a_{1}=S(z)$ is the scalar curvature of $\omega$ and each $a_{j}(z)$ is a polynomial of curvature and its covariant derivative. Thus we get the following complete asymptotics of $E_{m m}^{N}(z)$ :

$$
\begin{aligned}
E_{m m}^{N}(z) & =\left(\frac{\omega}{\pi} N+\frac{i \partial \bar{\partial} S(z)}{\pi} N^{-1}+\cdots\right)^{m} \\
& =N^{m}\left(\frac{\omega}{\pi}\right)^{m}+m N^{m-2}\left(\frac{\omega}{\pi}\right)^{m-1} \wedge \frac{i \partial \bar{\partial} S(z)}{\pi}+\cdots,
\end{aligned}
$$

which implies that the integration (51) admits complete asymptotics. Each $a_{j}(h, s)$ is well defined since $G_{s} \in L^{1}(M \times M)$ for $0 \leq s<2 m$.

We can in fact compute each coefficient from the asymptotic expansion of $E_{m m}^{N}$. From the complete expansion of (54) and the identity

$$
m(i \partial \bar{\partial} S) \wedge \omega^{m-1}=\left(\Delta_{\omega} S\right) \omega^{m}
$$

we obtain (19).

Now we are ready to prove Theorem 1 . 
3.1. The case of $0<s<2 m$. We first prove the case $0<s<2 m$, the logarithm case when $s=0$ can be derived by modifications. By the definition of the discrete random Riesz energy, we have

$$
\begin{aligned}
E_{\mu_{h}^{N}} \mathcal{E}_{s} & =E_{\mu_{h}^{N}}\left(G_{s}(z, w), Z_{s_{1}^{N}, \cdots, s_{m}^{N}}(z) \otimes Z_{s_{1}^{N}, \cdots, s_{m}^{N}}(w)-Z_{\Delta}(z)\right) \\
& =\left(G_{s}(z, w), K_{m m}^{N}(z, w)-[\Delta] \wedge\left(E_{m m}^{N}(z) \otimes 1\right)\right) \\
& =\int_{M \times M} G_{s}(z, w)\left[K_{m m}^{N}(z, w)-[\Delta] \wedge\left(E_{m m}^{N}(z) \otimes 1\right)\right] \\
& =\int_{M} \int_{r_{g}(z, w) \leq \frac{b \sqrt{\log N}}{\sqrt{N}}} G_{s}(z, w)\left[K_{m m}^{N}(z, w)-[\Delta] \wedge\left(E_{m m}^{N}(z) \otimes 1\right)\right] \\
& +\int_{M} \int_{r_{g}(z, w) \geq \frac{b \sqrt{\log N}}{\sqrt{N}}} G_{s}(z, w) K_{m m}^{N}(z, w) .
\end{aligned}
$$

By Lemma 1, we rewrite $E_{\mu_{h}^{N}} \mathcal{E}_{s}$ as

$$
\begin{aligned}
E_{\mu_{h}^{N}} \mathcal{E}_{s} & =\int_{M} \int_{r_{g}(z, w) \leq \frac{b \sqrt{\log N}}{\sqrt{N}}} G_{s}(z, w)\left[K_{m m}^{N}(z, w)-[\Delta] \wedge\left(E_{m m}^{N}(z) \otimes 1\right)\right] \\
& +\int_{M} \int_{r_{g}(z, w) \geq \frac{b \sqrt{\log N}}{\sqrt{N}}} G_{s}(z, w) K_{m m}^{N}(z, w) \\
& -\int_{M \times M} G_{s}(z, w) E_{m m}^{N}(z) \wedge E_{m m}^{N}(w) \\
& +a_{1}(h, s) N^{2 m}+\sum_{j=2}^{p-1} a_{j}(h, s) N^{2 m-j}+O\left(N^{2 m-p}\right) \\
& =\int_{M} \int_{r_{g}(z, w) \leq \frac{b \sqrt{\log N}}{\sqrt{N}}} G_{s}(z, w)\left[K_{m m}^{N}(z, w)-[\Delta] \wedge\left(E_{m m}^{N}(z) \otimes 1\right)\right. \\
& \left.-E_{m m}^{N}(z) \wedge E_{m m}^{N}(w)\right] \\
& +\int_{M} \int_{r_{g}(z, w) \geq \frac{b \sqrt{\log N}}{\sqrt{N}}} G_{s}(z, w)\left[K_{m m}^{N}(z, w)-E_{m m}^{N}(z) \wedge E_{m m}^{N}(w)\right] \\
& +a_{1}(h, s) N^{2 m}+\sum_{j=2}^{p-1} a_{j}(h, s) N^{2 m-j}+O\left(N^{2 m-p}\right) \\
& :=I+I I+I I I,
\end{aligned}
$$

where $p$ is a positive integer which will be chosen later on.

The following lemma asserts that $I I$ could be ignored.

\section{Lemma 2.}

$$
I I=O\left(N^{-q+\frac{s}{2}}\right),
$$

where $q$ is a positive integer which can be chosen large enough.

Proof. If $r_{g}(z, w) \geq \frac{b \sqrt{\log N}}{\sqrt{N}}$, by the definition of Riesz functions, we have

$$
\left|G_{s}(z, w)\right| \leq c\left(\frac{N}{\log N}\right)^{\frac{s}{2}}
$$


as $N$ large enough. If we choose $k=m$ in the estimate (43),

$$
K_{m m}^{N}(z, w)-E_{m m}^{N}(z) \wedge E_{m m}^{N}(w)=O\left(N^{-q}\right) \text { on } r_{g}(z, w) \geq \frac{b \sqrt{\log N}}{\sqrt{N}}
$$

where $b>\sqrt{q+2 k+3}$. If we combine these two estimate, the term $I I$ reads

$$
\int_{M} \int_{r_{g}(z, w) \geq \frac{b \sqrt{\log N}}{\sqrt{N}}} G_{s}(z, w)\left[K_{m m}^{N}(z, w)-E_{m m}^{N}(z) \wedge E_{m m}^{N}(w)\right]=O\left(N^{-q+\frac{s}{2}}\right),
$$

which completes the proof.

Now we turn to estimate $I$ to finish the proof of Theorem 1 ,

\section{Lemma 3.}

$$
I=c_{m}(s) N^{m+\frac{s}{2}}+O\left(N^{m+\frac{s-1}{2}}(\log N)^{m-\frac{s}{2}}\right) \quad 0<s<2 m, s<4,
$$

where $c(s)$ is a constant given explicitly in the proof.

Proof. We change variable

$$
w=z+\frac{u}{\sqrt{N}} .
$$

Then by the rescaling property of (44), we have

$$
\begin{aligned}
& K_{m m}^{N}(z, w)-[\Delta] \wedge\left(E_{m m}^{N}(z) \otimes 1\right) \\
& \quad=K_{m m}^{N}\left(z, z+\frac{u}{\sqrt{N}}\right)-[\Delta] \wedge\left(E_{m m}^{N}(z) \otimes 1\right) \\
& =N^{m} \kappa_{m m}(|u|)\left(\frac{\omega}{\pi}\right)^{m} \wedge\left(\frac{i}{2 \pi} \partial \bar{\partial}|u|^{2}\right)^{m}+O\left(N^{m-\frac{1}{2}}\right),
\end{aligned}
$$

where $\kappa_{m m}(|u|)$ is defined in (45) with $k=m$.

By the estimate of (54),

$$
E_{m m}^{N}(z) \wedge E_{m m}^{N}(w)=N^{2 m}\left(\frac{\omega(z)}{\pi}\right)^{m} \wedge\left(\frac{\omega(w)}{\pi}\right)^{m}+O\left(N^{2 m-2}\right) .
$$

By choosing the normal coordinate, if $\varphi$ is the Kähler potential, then we have

$$
\varphi\left(z+\frac{u}{\sqrt{N}}\right)=\frac{|u|^{2}}{2 N}+O\left(\frac{|u|^{3}}{N^{\frac{3}{2}}}\right) .
$$

Since $u=\sqrt{N}(w-z)$, we have

$$
\partial_{w} \bar{\partial}_{w} \varphi(w)=\partial_{u} \bar{\partial}_{u} \varphi\left(z+\frac{u}{\sqrt{N}}\right)=\partial_{u} \bar{\partial}_{u} \frac{|u|^{2}}{2 N}+O\left(N^{-\frac{3}{2}}\right) .
$$

Therefore,

$$
\frac{N}{\pi} \omega(w)=\frac{i}{2 \pi} \partial_{u} \bar{\partial}_{u}|u|^{2}+O\left(N^{-\frac{1}{2}}\right) .
$$

Thus we rewrite (56) near the diagonal when $|u|_{g} \leq b \sqrt{\log N}$ as

$$
E_{m m}^{N}(z) \wedge E_{m m}^{N}\left(z+\frac{u}{\sqrt{N}}\right)=N^{m}\left(\frac{\omega}{\pi}\right)^{m} \wedge\left(\frac{i}{2 \pi} \partial \bar{\partial}|u|^{2}\right)^{m}+O\left(N^{m-\frac{1}{2}}\right) .
$$


Recall the definition of $I$,

$$
\begin{aligned}
& I=\int_{M} \int_{r_{g}(z, w) \leq \frac{b \sqrt{\log N}}{\sqrt{N}}} G_{s}(z, w)\left[K_{m m}^{N}(z, w)-[\Delta] \wedge\left(E_{m m}^{N}(z) \otimes 1\right)\right. \\
& \left.-E_{m m}^{N}(z) \wedge E_{m m}^{N}(w)\right]
\end{aligned}
$$

Now we apply identities (55), (58) to rewrite it as

$$
\begin{gathered}
I=N^{m} \int_{M} \int_{0<|u|_{g} \leq b \sqrt{\log N}} G_{s}\left(z, z+\frac{u}{\sqrt{N}}\right)\left[\kappa_{m m}(|u|)-1+O\left(N^{-\frac{1}{2}}\right)\right] \\
\cdot\left(\frac{\omega}{\pi}\right)^{m} \wedge\left(\frac{i}{2 \pi} \partial \bar{\partial}|u|^{2}\right)^{m} \cdot
\end{gathered}
$$

The error term $O\left(N^{-\frac{1}{2}}\right)$ comes from estimate (55) and (58).

Thus we further rewrite (59) as

$$
\begin{array}{r}
I=N^{m} \int_{M} \int_{|u|_{g} \leq b \sqrt{\log N}} G_{s}\left(z, z+\frac{u}{\sqrt{N}}\right)\left[\kappa_{m m}(|u|)-1\right] \\
\cdot\left(\frac{\omega}{\pi}\right)^{m} \wedge\left(\frac{i}{2 \pi} \partial \bar{\partial}|u|^{2}\right)^{m}+R_{s} .
\end{array}
$$

Denote $I=\tilde{I}+R_{s}$. The estimate of the error term $R_{s}$ is as follows:

$$
R_{s}=O\left(N^{m-\frac{1}{2}}\right) \int_{M} \int_{|u|_{g} \leq b \sqrt{\log N}} G_{s}\left(z, z+\frac{u}{\sqrt{N}}\right)\left(\frac{\omega}{\pi}\right)^{m} \wedge\left(\frac{i}{2 \pi} \partial \bar{\partial}|u|^{2}\right)^{m} .
$$

If we choose the polar coordinate, we have

$$
\begin{aligned}
& \left|\int_{M} \int_{0 \leq|u|_{g} \leq b \sqrt{\log N}} G_{s}\left(z, z+\frac{u}{\sqrt{N}}\right)\left(\frac{\omega}{\pi}\right)^{m} \wedge\left(\frac{i}{2 \pi} \partial \bar{\partial}|u|^{2}\right)^{m}\right| \\
& \leq c \int_{0 \leq|u|_{g} \leq b \sqrt{\log N}} \frac{1}{\left|\frac{u}{\sqrt{N}}\right| s}|u|^{2 m-1} d|u| \leq c N^{\frac{s}{2}}(\log N)^{m-\frac{s}{2}} .
\end{aligned}
$$

In the last step, we apply the estimate of the geodesic distance function $r_{g}(z, w)$ near the origin:

$$
r_{g}\left(z, z+\frac{u}{\sqrt{N}}\right)=\frac{|u|}{\sqrt{N}}+O\left(N^{-\frac{3}{2}}\right)
$$

in the normal coordinate. Thus,

$$
R_{s}=O\left(N^{m+\frac{s-1}{2}}(\log N)^{m-\frac{s}{2}}\right) \text { if } 0<s<2 m .
$$

The estimate of the integration $\tilde{I}$ is as follows. First we rewrite it as

$$
\begin{aligned}
\tilde{I}= & N^{m+\frac{s}{2}} \int_{M} \int_{0 \leq|u| \leq \infty} \frac{1}{|u|^{s}}\left[\kappa_{m m}(|u|)-1\right]\left(\frac{\omega}{\pi}\right)^{m} \wedge\left(\frac{i}{2 \pi} \partial \bar{\partial}|u|^{2}\right)^{m} \\
& + \text { lower order terms } \\
:= & A_{s}+B_{s}
\end{aligned}
$$


by applying the expansion of the geodesic distance function (61). We use polar coordinates to compute the leading term,

$$
\begin{aligned}
A_{s} & =N^{m+\frac{s}{2}} \int_{M} \int_{0 \leq|u| \leq \infty} \frac{1}{|u|^{s}}\left[\kappa_{m m}(|u|)-1\right]\left(\frac{\omega}{\pi}\right)^{m} \wedge\left(\frac{i}{2 \pi} \partial \bar{\partial}|u|^{2}\right)^{m} \\
& =N^{m+\frac{s}{2}} \frac{V_{2 m-1}}{\pi^{m}} \int_{0 \leq|u| \leq \infty} \frac{1}{|u|^{s}}\left[\kappa_{m m}(|u|)-1\right]|u|^{2 m-1} d|u| \\
& =\left(2 m \int_{0 \leq|u| \leq \infty}\left[\kappa_{m m}(r)-1\right] r^{2 m-1-s} d r\right) N^{m+\frac{s}{2}} \\
& :=c_{m}(s) N^{m+\frac{s}{2}},
\end{aligned}
$$

where $V_{2 m-1}$ is the volume of the $(2 m-1)$-dimensional unit sphere.

The integrand in $c_{m}(s)$ is always integrable on the tail $a<|u|<\infty$ as a large enough. This is easy to see because of the asymptotic property $\kappa_{m m}(r)=1+$ $O\left(r^{4} e^{-r^{2}}\right)$ as $r \rightarrow \infty$. But we need some conditions to ensure $c_{m}(s)$ to be well defined near the origin. Since we know $\kappa_{m m}(r) \rightarrow \frac{m+1}{4} r^{4-2 m}$ as $r \rightarrow 0$, it's easy to check that $s<4$ is a necessary condition.

Moreover, we have the easy estimate

$$
B_{s}=O\left(N^{m+\frac{s}{2}-1}\right) .
$$

Thus the growth of the error term $R_{s}$ will dominate the growth of $B_{s}$, and

$$
I=A_{s}+R_{s}=c_{m}(s) N^{m+\frac{s}{2}}+O\left(N^{m+\frac{s-1}{2}}(\log N)^{m-\frac{s}{2}}\right),
$$

which completes the proof.

We now finish the proof of the main result of Theorem 1 for $0<s<2 m$.

Proof. For the case $0<s<2 m$ and $s<4$, recall the decomposition of $E_{\mu_{h}^{N}} \mathcal{E}_{s}$ :

$$
E_{\mu_{h}^{N}} \mathcal{E}_{s}=a_{1}(h, s) N^{2 m}+\sum_{j=2}^{p-1} a_{j}(h, s) N^{2 m-j}+O\left(N^{2 m-p}\right)+I+I I .
$$

Now we apply the estimates in Lemma 2 and Lemma 3. We have

$$
\begin{aligned}
E_{\mu_{h}^{N}} \mathcal{E}_{s}= & a_{1}(h, s) N^{2 m}+\sum_{j=2}^{p-1} a_{j}(h, s) N^{2 m-j}+O\left(N^{2 m-p}\right) \\
& +c_{m}(s) N^{m+\frac{s}{2}}+O\left(N^{m+\frac{s-1}{2}}(\log N)^{m-\frac{s}{2}}\right)+O\left(N^{-q+\frac{s}{2}}\right) .
\end{aligned}
$$

The smallest integer $p$ such that $2 m-(p-1)>m+\frac{s}{2}$ will ensure the above expansion is well defined, i.e., $p=\left[m-\frac{s}{2}\right]+1$. The last term is negligible for $q$ large enough. Thus we rewrite it as

$$
\begin{aligned}
E_{\mu_{h}^{N}} \mathcal{E}_{s}= & a_{1}(h, s) N^{2 m}+\sum_{j=2}^{p-1} a_{j}(h, s) N^{2 m-j} \\
& +c_{m}(s) N^{m+\frac{s}{2}}+O\left(N^{m+\frac{s-1}{2}}(\log N)^{m-\frac{s}{2}}\right),
\end{aligned}
$$

which completes the proof. 
3.2. The case of $s=0$. Now we sketch the proof for the case of $s=0$ in Theorem 1

First, as before, we rewrite it as

$$
\begin{aligned}
E_{\mu_{h}^{N}} \mathcal{E}_{0} & =-\int_{M} \int_{r_{g}(z, w) \leq \frac{b \sqrt{\log N}}{\sqrt{N}}} \log r_{g}(z, w)\left[K_{m m}^{N}(z, w)-[\Delta] \wedge\left(E_{m m}^{N}(z) \otimes 1\right)\right. \\
& -\int_{M} \int_{r_{g}(z, w) \geq \frac{b \sqrt{\log N}}{\sqrt{N}}} \log r_{g}(z, w)\left[K_{m m}^{N}(z, w)-E_{m m}^{N}(z) \wedge E_{m m}^{N}(w)\right] \\
& +a_{1}(h, 0) N^{2 m}+\sum_{j=2}^{N-1} a_{j}(h, 0) N^{2 m-j}+O\left(N^{2 m-p}\right) \\
& :=I+I I+I I I .
\end{aligned}
$$

Follow the steps in Lemma 2

$$
I I=O\left(N^{-q} \log N\right) \text { for } q \text { large enough }
$$

since $\left|G_{0}\right|$ is bounded by $\log N$ if $r_{g}(z, w) \geq \frac{b \sqrt{\log N}}{\sqrt{N}}$. Thus the term $I I$ can be ignored in the final expansion.

The statement in Theorem 1 about logarithmic Riesz energies follows directly by the following lemma.

\section{Lemma 4.}

$$
I=-N^{m} \log \sqrt{N}-c_{m} N^{m}+O\left(N^{m-\frac{1}{2}}(\log N)^{m+1}\right),
$$

where $c$ is a constant given by (17).

Proof. Following the proof of (60) in Lemma 3, we rewrite $I=\tilde{I}+R_{0}$ as follows:

$$
\begin{array}{r}
I=N^{m} \int_{M} \int_{|u|_{g} \leq b \sqrt{\log N}} G_{0}\left(z, z+\frac{u}{\sqrt{N}}\right)\left[\kappa_{m m}(|u|)-1\right] \\
\cdot\left(\frac{\omega}{\pi}\right)^{m} \wedge\left(\frac{i}{2 \pi} \partial \bar{\partial}|u|^{2}\right)^{m}+R_{0},
\end{array}
$$

where

$$
R_{0}=O\left(N^{m-\frac{1}{2}}\right) \int_{M} \int_{|u|_{g} \leq b \sqrt{\log N}} G_{0}\left(z, z+\frac{u}{\sqrt{N}}\right)\left(\frac{\omega}{\pi}\right)^{m} \wedge\left(\frac{i}{2 \pi} \partial \bar{\partial}|u|^{2}\right)^{m} .
$$

Using the geodesic distance estimate (61), we have

$$
\left.\left|\int_{0 \leq|u|_{g} \leq b \sqrt{\log N}} \log \left(\frac{|u|_{g}}{\sqrt{N}}\right)\right| u\right|^{2 m-1} d|u| \mid \leq c(\log N)^{m+1} .
$$

Thus,

$$
R_{0}=O\left(N^{m-\frac{1}{2}}(\log N)^{m+1}\right) \text { if } s=0 .
$$


We further rewrite $\tilde{I}=A_{0}+B_{0}$ by applying the expansion of the geodesic distance function (61) again:

$$
\begin{aligned}
A_{0}= & -N^{m} \int_{M} \int_{0 \leq|u| \leq \infty} \log \left(\frac{|u|}{\sqrt{N}}\right)\left[\kappa_{m m}(|u|)-1\right]\left(\frac{\omega}{\pi}\right)^{m} \wedge\left(\frac{i}{2 \pi} \partial \bar{\partial}|u|^{2}\right)^{m} \\
= & N^{m} \log \sqrt{N} \int_{0 \leq|u| \leq \infty}\left[\kappa_{m m}(|u|)-1\right]\left(\frac{i}{2 \pi} \partial \bar{\partial}|u|^{2}\right)^{m} \\
& -\left(2 m \int_{0 \leq|u| \leq \infty} \log r\left[\kappa_{m m}(r)-1\right] r^{2 m-1} d r\right) N^{m}
\end{aligned}
$$

Now claim that

$$
\int_{0 \leq|u| \leq \infty}\left[\kappa_{m m}(|u|)-1\right]\left(\frac{i}{2 \pi} \partial \bar{\partial}|u|^{2}\right)^{m}=-1 .
$$

We calculate the integration directly by the rescaling property:

$$
\begin{aligned}
& \int_{M}\left(\frac{\omega}{\pi}\right)^{m} \int_{0 \leq|u| \leq \infty}\left[\kappa_{m m}(|u|)-1\right]\left(\frac{i}{2 \pi} \partial \bar{\partial}|u|^{2}\right)^{m} \\
& =\lim _{N \rightarrow \infty} \frac{1}{N^{m}} \int_{M} \int_{r_{g}(z, w) \leq \frac{b \sqrt{\log N}}{\sqrt{N}}}\left\{K_{m m}^{N}(z, w)-[\Delta] \wedge E_{m m}^{N}(w)\right. \\
& =\lim _{N \rightarrow \infty} \frac{1}{N^{m}}\left(\int_{M} \int_{M} \cdots-\int_{M} \int_{r_{g}(z, w) \geq \frac{b \sqrt{\log N}}{\sqrt{N}}} \cdots\right) .
\end{aligned}
$$

The claim follows by the following two calculations: By the definition of correlation currents, the first term reads as

$$
\begin{aligned}
& \lim _{N \rightarrow \infty} \frac{1}{N^{m}} \int_{M} \int_{M}\left\{K_{m m}^{N}(z, w)-[\Delta] \wedge E_{m m}^{N}(w)-E_{m m}^{N}(z) \wedge E_{m m}^{N}(w)\right\} \\
= & \lim _{N \rightarrow \infty} \frac{1}{N^{m}}\left(N^{2 m}-N^{m}-N^{2 m}\right)=-1 .
\end{aligned}
$$

The second term reads as

$$
\begin{aligned}
& \lim _{N \rightarrow \infty} \frac{1}{N^{m}} \int_{M} \int_{r_{g}(z, w) \geq \frac{b \sqrt{\log N}}{\sqrt{N}}}\left(K_{m m}^{N}(z, w)-E_{m m}^{N}(z) \wedge E_{m m}^{N}(w)\right) \\
& =\lim _{N \rightarrow \infty} O\left(N^{-q-m}\right)=0,
\end{aligned}
$$

where we applied estimate (43) for $q$ large enough. Thus

$$
A_{0}=-N^{m} \log \sqrt{N}-c_{m} N^{m},
$$

where $c_{m}$ is given by (17). The integral above is also well defined by the asymptotic properties of $\kappa_{m m}(r)$. Also, it's easy to check that

$$
B_{0}=O\left(N^{m-1}\right) \text {. }
$$

The growth of $B_{0}$ is less than the growth of $R_{0}$; thus the error terms $B_{0}$ can be absorbed in the error term $R_{0}$. Thus in the case of $s=0$,

$$
I=A_{0}+R_{0}=-N^{m} \log \sqrt{N}-c_{m} N^{m}+O\left(N^{m-\frac{1}{2}}(\log N)^{m+1}\right),
$$

which completes the proof. 


\section{Proof of Proposition 1}

The proof is almost trivial but we include it to clarify the structure of $\kappa_{m m}(r)-1$ (46). The main point is that $c_{m}(s)$ is a meromorphic function with poles at $s=$ $\min 4,2 m$. When $m \geq 2$, the only pole for $s \in[0,4]$ is at $s=4$. When $m \geq 3$ the residue is positive, while for $m=2$ it is negative. When $m=1$ it also has a pole at $s=2 \in[0,4]$ with a negative residue. The proposition just uses these facts to draw conclusions on positivity close enough to the poles.

The results are illustrated in the following computer graphics in the cases $m=$ $2,3,4$. The graphs for $m \geq 4$ are rather similar to that for $m=3,4$. The integral in (71) or more precisely $\int_{0}^{\infty}\left(\kappa_{m m}-1\right) r^{2 m-1-s} d r$ is evaluated numerically over the interval $[0,10000]$ so that positivity of the numerical integration implies positivity of the true integral. As the figures show, $c_{m}(s)<0$ for small $s$, in which case the random zeros have smaller Riesz $s$-energy on average than general random point. When $m=2$ it appears to always be negative, so that random zeros have less energy than random points on average. When $m \geq 3, c_{m}(s)>0$ for $s$ somewhat larger than $s=1$, and the rise is very steep as $s \rightarrow 4$. As $m$ increases, the clumping of zeros increases and hence the Riesz energies increase.

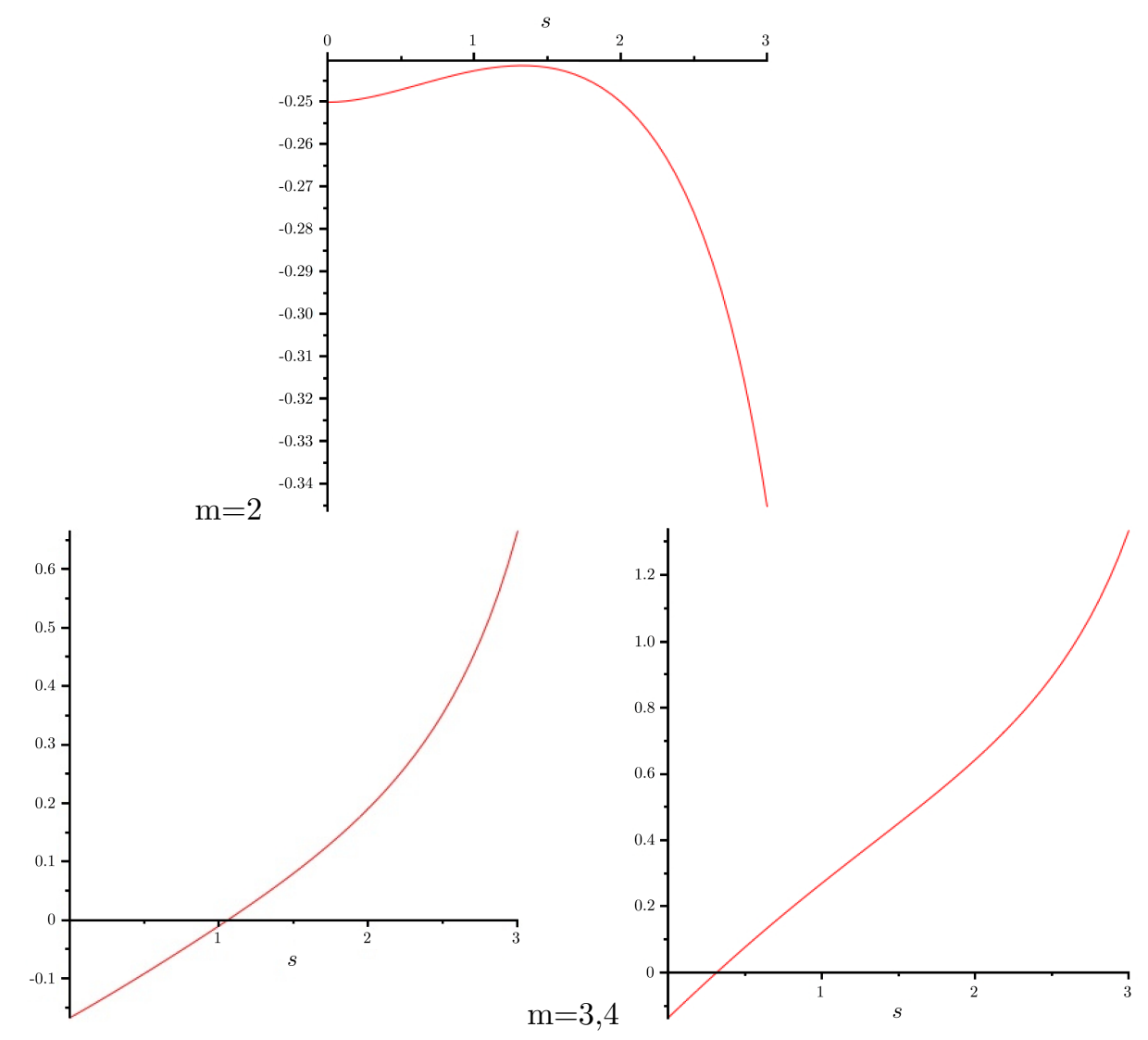


Here are the details: We observe that the numerator of $\kappa_{m m}$ consists of three terms,

$$
\left\{\begin{array}{l}
\kappa_{m m}^{I}(r)=m\left(1-v^{m+1}\right)(1-v), \\
\kappa_{m m}^{I I}(r)=r^{2}(2 m+2)\left(v^{m+1}-v\right), \\
\kappa_{m m}^{I I I}(r)=r^{4}\left[v^{m+1}+v^{m}+(\{m+1\} v+1)\left(v^{m}-v\right) /(v-1)\right] .
\end{array}\right.
$$

Since $v<1$ only the second is negative. Furthermore the second and third terms are divisible by $v=e^{-r^{2}}$, and therefore the first term dominates for large $r$. We claim that there exists $r_{0}(m)$ so that $\kappa_{m m}(r)-1$ is positive for $r \geq r_{0}(m)$. We do not attempt to find the best $r_{0}(m)$ but rather look for a simple one.

We first drop the terms $v^{m}$ or higher powers from the numerator, simplifying the numerator to

$$
N_{0}(m, x):=m\left(1-e^{-x}\right)-(2 m+2) x e^{-x}+x^{2}\left[(m+1) e^{-x}+1\right] \frac{e^{-x}}{1-e^{-x}}
$$

with $x=r^{2}$. We would like to find $x$ so that $N_{0}(m, x)>m\left(1-e^{-x}\right)^{m+2}$, i.e. so that

$$
x^{2}\left[\frac{(m+1)}{m} e^{-x}+\frac{1}{m}\right] \frac{e^{-x}}{1-e^{-x}}-\frac{(2 m+2)}{m} x e^{-x}-e^{-x}>\left(1-e^{-x}\right)^{m+2}-1 .
$$

In fact, the left side is easily seen to be positive if $x \geq(2 m+3)$. Returning to our original numerator, we want to show that $N_{0}(m, x)>m$ if $x \geq(2 m+3)$. Given the above observations, it suffices to show that the missing terms $-m v^{m+1}(1-v)+$ $r^{2}(2 m+2) v^{m+1}>0$, and this also holds when $x \geq 3$. Consequently, $\kappa_{m m}(r)-1>0$ if $x \geq(2 m+3)$. Thus, we can take $r_{0}(m)=\sqrt{(2 m+3)}$. Hence, to prove that $c_{m}(s)>0$ it suffices to show that

$$
\int_{0}^{\sqrt{2 m+3}}\left[\kappa_{m m}(r)-1\right] r^{2 m-1-s} d r>0
$$

for $s \geq s_{m}$. Indeed, the contribution for small $r$ has the form

$$
\int_{0}^{\epsilon}\left[\kappa_{m m}(r)-1\right] r^{2 m-1-s} d r=\frac{m+1}{4} \frac{\epsilon^{4-s}}{4-s}-\frac{\epsilon^{2 m-s}}{2 m-s}+O_{m}\left(\epsilon^{8-s}\right),
$$

and only the first term has a pole as $s \rightarrow 4^{-}$when $m \geq 3$. When $m=2$ the integral of -1 also blows up at $s=4$ and the coefficient of $\frac{1}{4-s}$ is $-1 / 4$, so the integral is negative for $s$ near 4 ; when $m=1$ the integral of the -1 term tends to $-\infty$ at $s=2$. Hence in proving positivity we assume $m \geq 3$. On the other hand, for $m \geq 3$ the integral on the left side of (71) over the interval $[\epsilon, \sqrt{2 m+3}]$ is continuous in $s \in[0,4]$ and of course has a uniform upper bound. Hence (171) holds for $s<4$ sufficiently close to 4 .

\section{Proof of Theorem 2}

The proof of Theorem 2 is similar to Theorem 11. For simplicity, we only prove the case when $s \neq 0$; the result for logarithm energies can be derived by a few modifications. We now sketch the proof as follows. 
Proof. Suppose $Z_{s_{1}^{N}, \cdots, s_{k}^{N}}$ is the zero locus of $k<m$ holomorphic random sections, i.e., the analytic subvariety of positive dimension $m-k$. By the definition of the continuous random Riesz energy (21) and (22), we have

$$
\begin{aligned}
E_{\mu_{h}^{N}} \mathcal{E}_{s}^{N}= & E\left(G_{s}(z, w) d V_{k}(z) d V_{k}(w), Z_{s_{1}^{N}, \cdots, s_{k}^{N}}(z) \otimes Z_{s_{1}^{N}, \cdots, s_{k}^{N}}(w)\right) \\
= & \int_{M \times M} G_{s}(z, w) d V_{k}(z) d V_{k}(w) \wedge K_{k m}^{N}(z, w) \\
= & \int_{M \times M} G_{s}(z, w) d V_{k}(z) d V_{k}(w) \wedge E_{k m}^{N}(z) \wedge E_{k m}^{N}(w) \\
& +\int_{M \times M} G_{s}(z, w) d V_{k}(z) d V_{k}(w) \wedge\left[K_{k m}^{N}(z, w)-E_{k m}^{N}(z) \wedge E_{k m}^{N}(w)\right] \\
= & : I+I I,
\end{aligned}
$$

where $d V_{k}=\frac{\omega^{m-k}}{(m-k) !}$.

Following the step in Lemma 1 $E_{k m}^{N}(z)$, which is the expectation of zero locus $Z_{s_{1}^{N}, \cdots, s_{k}^{N}}$, admits the following expansions:

$$
\begin{aligned}
E_{k m}^{N}(z) & =\left(\frac{\omega}{\pi} N+\frac{i \partial \bar{\partial} S(z)}{\pi} N^{-1}+\cdots\right)^{k} \\
& =N^{k}\left(\frac{\omega}{\pi}\right)^{k}+k N^{k-2}\left(\frac{\omega}{\pi}\right)^{k-1} \wedge \frac{i \partial \bar{\partial} S(z)}{\pi}+\cdots
\end{aligned}
$$

Thus, it's easy to get

$I=\left(\frac{\pi^{m-k}}{(m-k) !}\right)^{2}\left[a_{1}(h, s) N^{2 k}+w_{2}(k) a_{2}(h, s) N^{2 k-2}+w_{3}(k) a_{3}(h, s) N^{2 k-3}+\cdots\right]$,

where all $a_{j}$ are the same as in Lemma 1 and $w_{j}$ is the weight of $a_{j}$, in particular, $w_{2}=\frac{k}{m}$.

To the second term $I I$, we need to decompose it to be

$$
\begin{aligned}
I I & =\int_{M} \int_{r_{g}(z, w) \leq \frac{b \log \sqrt{N}}{\sqrt{N}}} \cdots+\int_{M} \int_{r_{g}(z, w) \geq \frac{b \log \sqrt{N}}{\sqrt{N}}} \cdots \\
& =: I I I+I V .
\end{aligned}
$$

Recall the estimate (43):

$$
K_{k m}^{N}(z, w)-E_{k m}^{N}(z) \wedge E_{k m}^{N}(w)=O\left(N^{-q}\right) \text { on } r_{g}(z, w) \geq \frac{b \sqrt{\log N}}{\sqrt{N}} .
$$

This is true for any $k$. Thus, following the argument in Lemma 2 ,

$$
I V=O\left(N^{-q+\frac{s}{2}}\right)
$$

for $q$ large enough. Thus $I V$ can be ignored in the final expansion of $E_{\mu_{h}^{N}} \mathcal{E}_{s}$. Thus we will finish the proof if we can get the estimate of $I I I$.

We change variables,

$$
w=z+\frac{u}{\sqrt{N}} .
$$

Then by the rescaling property of (44), we have

$$
K_{k m}^{N}\left(z, z+\frac{u}{\sqrt{N}}\right)=N^{k} \kappa_{k m}(|u|)\left(\frac{\omega}{\pi}\right)^{k} \wedge\left(\frac{i}{2 \pi} \partial \bar{\partial}|u|^{2}\right)^{k}+O\left(N^{k-\frac{1}{2}}\right) .
$$


Following the step in proving the estimate (58), we have

$$
E_{k m}^{N}(z) \wedge E_{k m}^{N}\left(z+\frac{u}{\sqrt{N}}\right)=N^{k}\left(\frac{\omega}{\pi}\right)^{k} \wedge\left(\frac{i}{2 \pi} \partial \bar{\partial}|u|^{2}\right)^{k}+O\left(N^{k-\frac{1}{2}}\right) .
$$

Thus we further rewrite $I I I$ as

$$
\begin{array}{r}
I I I=N^{2 k-m}\left(\frac{\pi^{m-k}}{(m-k) !}\right)^{2} \int_{M} \int_{|u|_{g} \leq b \sqrt{\log N}} G_{s}\left(z, z+\frac{u}{\sqrt{N}}\right)\left[\kappa_{k m}(|u|)-1\right] \\
\cdot\left(\frac{\omega}{\pi}\right)^{m} \wedge\left(\frac{i}{2 \pi} \partial \bar{\partial}|u|^{2}\right)^{m}+R_{s},
\end{array}
$$

where

$$
R_{s}=O\left(N^{2 k-m-\frac{1}{2}}\right) \int_{M} \int_{|u|_{g} \leq b \sqrt{\log N}} G_{s}\left(z, z+\frac{u}{\sqrt{N}}\right)\left(\frac{\omega}{\pi}\right)^{m} \wedge\left(\frac{i}{2 \pi} \partial \bar{\partial}|u|^{2}\right)^{m} .
$$

Thus by the same argument as in Lemma 3, we have

$$
R_{s}=O\left(N^{2 k-m+\frac{s-1}{2}}(\log N)^{m-\frac{s}{2}}\right) \text { if } 0<s<2 m .
$$

Applying the asymptotics of geodesic distance function (61) near the origin, we rewrite it as

$$
\begin{aligned}
I I I= & N^{2 k-m+\frac{s}{2}}\left(\frac{\pi^{m-k}}{(m-k) !}\right)^{2} \int_{M} \int_{0 \leq|u| \leq \infty} \frac{1}{|u|^{s}}\left[\kappa_{k m}(|u|)-1\right]\left(\frac{\omega}{\pi}\right)^{m} \wedge\left(\frac{i}{2 \pi} \partial \bar{\partial}|u|^{2}\right)^{m} \\
& +R_{s}+\text { lower order terms } \\
= & N^{2 k-m+\frac{s}{2}}\left(\frac{\pi^{m-k}}{(m-k) !}\right)^{2} \frac{V_{2 m-1}}{\pi^{m}} \int_{0 \leq|u| \leq \infty} \frac{1}{|u|^{s}}\left[\kappa_{k m}(|u|)-1\right]|u|^{2 m-1} d|u| \\
& +R_{s}+\text { lower order terms } \\
= & : d_{m}(s, k) N^{2 k-m+\frac{s}{2}}+R_{s}+\text { lower order terms }
\end{aligned}
$$

by polar coordinate.

The integrand in $d(s, k)$ is always integrable on the tail $a<|u|<\infty$ as $a$ large enough because of the asymptotic property $\kappa_{k m}(r)=1+O\left(r^{4} e^{-r^{2}}\right)$ as $r \rightarrow \infty$. To ensure $d_{m}(s, k)$ to be well defined near the origin, recall that when $k \neq m$,

$$
\kappa_{k m}(r) \sim r^{-2 k} \text { as } r \rightarrow 0
$$

hence, $s<2(m-k)$ is a necessary condition.

Now we can turn to prove Theorem 2.

Proof. For the case $s \neq 0, s<2(m-k)$, recall the decomposition of $E_{\mu_{h}^{N}} \mathcal{E}_{s}$ :

$$
\begin{aligned}
E_{\mu_{h}^{N}} \mathcal{E}_{s}= & I+I I \\
= & \left(\frac{\pi^{m-k}}{(m-k) !}\right)^{2}\left[a_{1}(h, s) N^{2 k}+w_{2}(k) a_{2}(h, s) N^{2 k-2}+\cdots\right]+O\left(N^{2 k-p}\right) \\
& +d_{m}(s, k) N^{2 k-m+\frac{s}{2}}+O\left(N^{2 k-m+\frac{s-1}{2}}(\log N)^{m-\frac{s}{2}}\right)+O\left(N^{-q+\frac{s}{2}}\right) .
\end{aligned}
$$


The smallest integer $p$ such that $2 k-(p-1)>2 k-m+\frac{s}{2}$ will ensure the above expansion is well defined, i.e., $p=\left[m-\frac{s}{2}\right]+1$, which completes the proof. If we further choose $q$ large enough, we obtain

$$
\begin{aligned}
E_{\mu_{h}^{N}} \mathcal{E}_{s}= & \left(\frac{\pi^{m-k}}{(m-k) !}\right)^{2}\left[a_{1}(h, s) N^{2 k}+w_{2}(k) a_{2}(h, s) N^{2 k-2}+\cdots\right] \\
& +d_{m}(s, k) N^{2 k-m+\frac{s}{2}}+O\left(N^{2 k-m+\frac{s-1}{2}}(\log N)^{m-\frac{s}{2}}\right) .
\end{aligned}
$$

The same method is also applied to logarithm energies when $s=0$, but we omit the proof here.

\section{ACKNOWLEDGEMENTS}

Finally, we thank B. Shiffman for helpful comments and for the computer graphics. We also thank the referee for helpful comments.

\section{REFERENCES}

[ABS] D. Armentano, C. Beltran and M.Shub, Minimizing the discrete logarithmic energy on the sphere: The role of random polynomials, Trans. Amer. Math. Soc. 363 (6) (2011) 2955-2965. MR2775794 (2012f:31009)

[B] J.S. Brauchart, About the second term of the asymptotics for optimal Riesz energy on the sphere in the potential-theoretical case, Integral Transforms Spec. Funct. 17 (2006), no. 5, 321-328. MR2237493 (2007f:31011)

[BBGKS] B. Ballinger, G. Blekherman, H. Cohn, N. Giansiracusa, E. Kelly, and Achill Schrmann, Experimental study of energy-minimizing point configurations on spheres, Experimental Mathematics 18 (2009), 257-283. MR2555698(2010j:52037)

[BBP] B. Bergersen, D. Boal and P. Palffy-Muhoray, Equilibrium configurations of particles on a sphere: The case of logarithmic interaction, J. Phys. A: Math. Gen. 27 (1994) 2579-2586.

[BBWN] R. Berman, S. Boucksom and D. Witt-Nystrom, Fekete points and convergence towards equilibrium measures on complex manifolds, Acta Math. 207 (2011), no. 1, 1-27. MR.2863909 (2012j:32036)

[BSZ1] P. Bleher, B. Shiffman and S. Zelditch, Poincaré-Lelong approach to universality and scaling of correlations between zeros, Comm. Math. Phys. 208 (2000), 771-785. MR.1736335 (2002j:32017)

[BSZ2] P. Bleher, B. Shiffman and S. Zelditch, Universality and scaling of correlations between zeros on complex manifolds, Invent. Math. 142 (2000), 351-395. MR.1794066 (2002f:32037)

[BSZ3] P. Bleher, B. Shiffman and S. Zelditch, Correlations between zeros and supersymmetry, Commun. Math. Phys. 224 (2001), 255-269. MR1868999(2003d:32022)

[CK] H. Cohn and A. Kumar, Universally optimal distribution of points on spheres, J. Amer. Math. Soc. 20 (2007), 99-148. MR2257398(2007h:52009)

[GH] G. Griffiths and J. Harris, Principles of Algebraic Geometry, Wiley-Interscience (1978). MR.507725 (80b:14001)

[H] J. H. Hannay, Chaotic analytic zero points: Exact statistics for those of a random spin state, J. Phys. A 29 (1996), 314-320. MR1383056 (97a:82007)

[Hr] P. Hriljac Splitting fields of principal homogeneous spaces, Number Theory Seminar, Lect. Notes in Math. 1240, Springer-Verlag, 1987, pp. 214-229. MR894513 (88e:11043)

[HS] D. P. Hardin and E. B. Saff, Discretizing manifolds via minimum energy points, Notices Amer. Math. Soc. 51 (2004), 1186-1194. MR2104914 (2006a:41049)

[HiS] M. Hindry and J. H. Silverman, Sur le nombre de points de torsion rationnels sur une courbe elliptique [On the number of rational torsion points on an elliptic curve], C. R. Acad. Sci. Paris Sr. I Math. 329 (1999), no. 2, 97-100. MR1710502 (2000g:11047) 
[KS] A.B.J. Kuijlaars and E.B. Saff, Asymptotics for minimal discrete energy on the sphere, Trans. Amer. Math. Soc. 350 (2) (1998) 523-538. MR.1458327 (98e:11092)

[La] S. Lang, Introduction to Arakelov theory, Springer-Verlag, New York, 1988. MR.969124 (89m:11059)

[L] Z. Lu, On the lower order terms of the asymptotic expansion of Zelditch, Amer. J. Math. 122(2), 2000, pp 235-273. MR1749048(2002d:32034)

[M] K. Mahler, An inequality for the discriminant of a polynomial, Michigan Math. J. 11 (1964) 257-262. MR0166188 (29:3465)

[MKS] T. W. Melnyk, O. Knop and W. R. Smith, Extremal arrangements of points and unit charges on a sphere: Equilibrium configurations revisited, Canad. J. Chem. 55 (1977), no. 10, 1745-1761. MR0444497 (56:2848)

[RSZ] E. A. Rakhmanov, E. B. Saff, and Y. M. Zhou, Minimal discrete energy on the sphere, Math. Res. Lett. 1 (1994), no. 6, 647-662. MR1306011 (96e:78011)

[SZ1] B. Shiffman and S. Zelditch, Number variance of random zeros on complex manifolds, Geom. Funct. Anal. Vol. 18 (2008), 1422-1475. MR2465693 (2009k:32019)

[SZ2] B. Shiffman and S. Zelditch, Distribution of zeros of random and quantum chaotic sections of positive line bundles, Comm. Math. Phys. 200 (1999), no. 3, 661-683. MR:1675133 (2001j:32018)

[W1] G. Wagner, On the means of distances on the surface of a sphere (lower bounds), Pacific J. Math. 144 (1990), 389-398. MR1061328 (91e:52014)

[W2] G. Wagner, On the means of distances on the surface of a sphere II (upper bounds), Pacific J. Math. 153 (1992), 381-396. MR1159518 (93b:52007)

[Ze] S. Zelditch, Szegö kernels and a theorem of Tian, IMRN 6 (1998), 317-331. MR.1616718 (99g:32055)

[ZZe] S. Zelditch and Q. Zhong, Addendum to "Energies of zeros of random sections on Riemann surfaces", Indiana Univ. Math. J. 57 (2008), no. 4, 1753-1780. MR2919745

[Z] Q. Zhong, Energies of zeros of random sections on Riemann surfaces, Indiana Univ. Math. J. 57 (2008), no. 4, 1753-1780. MR2440880(2009k:58051)

Department of Mathematics and Statistics, McGill University, Montreal, Quebec, CANADA H3A 0G4

E-mail address: renjie@math.mcgill.ca

Department of Mathematics, Northwestern University, Evanston, Illinois 60208

E-mail address: zelditch@math.northwestern.edu 